\section{This paper is published as part of a $P C C P$ themed issue on dynamic nuclear polarization}

\section{Guest Editors: Robert Griffin and Thomas Prisner}

High field dynamic nuclear polarization-the renaissance

R. G. Griffin and T. F. Prisner, Phys. Chem. Chem. Phys., 2010, 12, 5737

http://dx.doi.org/10.1039/c0cp90019b

The discovery and demonstration of dynamic nuclear polarization-a personal and historical account Charles P. Slichter, Phys. Chem. Chem. Phys., 2010, 12, 5741

http://dx.doi.org/10.1039/c003286g

Communications

High power pulsed dynamic nuclear polarisation at 94 $\mathrm{GHz}$

Robert I. Hunter, Paul A. S. Cruickshank, David R. Bolton, Peter C. Riedi and Graham M. Smith, Phys. Chem. Chem. Phys., 2010, 12, 5752

http://dx.doi.org/10.1039/c002251a

Papers

DNP enhanced NMR using a high-power $94 \mathrm{GHz}$ microwave source: a study of the TEMPOL radical in toluene

Eugeny V. Kryukov, Mark E. Newton, Kevin J. Pike, David R. Bolton, Radoslaw M. Kowalczyk, Andrew P. Howes, Mark E. Smith and Ray Dupree, Phys. Chem. Chem. Phys., 2010, 12,5757

http://dx.doi.org/10.1039/c003189e

Rapid sample injection for hyperpolarized NMR spectroscopy

Sean Bowen and Christian Hilty, Phys. Chem. Chem. Phys., 2010, 12, 5766

http://dx.doi.org/10.1039/c002316g

Slice-selective single scan proton COSY with dynamic nuclear polarisation

Rafal Panek, Josef Granwehr, James Leggett and Walter Köckenberger, Phys. Chem. Chem. Phys., 2010, 12, 5771 http://dx.doi.org/10.1039/c002710n

Prospects for sub-micron solid state nuclear magnetic resonance imaging with low-temperature dynamic nuclear polarization

Kent R. Thurber and Robert Tycko, Phys. Chem. Chem.

Phys., 2010, 12, 5779

http://dx.doi.org/10.1039/c0cp00157k

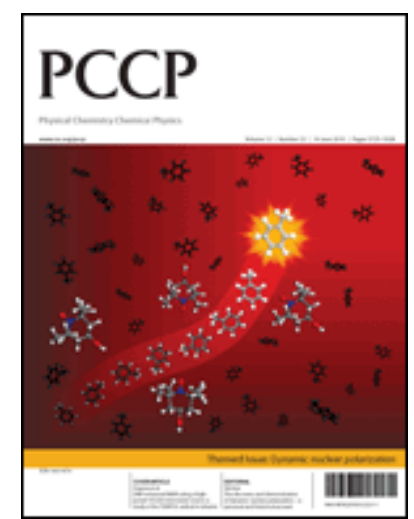

Liquid state DNP using a $260 \mathrm{GHz}$ high power gyrotron Vasyl Denysenkov, Mark J. Prandolini, Marat Gafurov, Deniz Sezer, Burkhard Endeward and Thomas F. Prisner, Phys. Chem. Chem. Phys., 2010, 12, 5786

http://dx.doi.org/10.1039/c003697h

Exploring the limits of electron-nuclear polarization transfer efficiency in three-spin systems

Nikolas Pomplun and Steffen J. Glaser, Phys. Chem. Chem. Phys., 2010, 12, 5791

http://dx.doi.org/10.1039/c003751f

Dynamic nuclear polarization experiments at $14.1 \mathrm{~T}$ for solid-state NMR

Yoh Matsuki, Hiroki Takahashi, Keisuke Ueda, Toshitaka Idehara, Isamu Ogawa, Mitsuru Toda, Hideo Akutsu and Toshimichi Fujiwara, Phys. Chem. Chem. Phys., 2010, 12, 5799

http://dx.doi.org/10.1039/c002268c

Trityl biradicals and ${ }^{13} \mathrm{C}$ dynamic nuclear polarization Sven Macholl, Haukur Jóhannesson and Jan Henrik Ardenkjaer-Larsen, Phys. Chem. Chem. Phys., 2010, 12 5804

http://dx.doi.org/10.1039/c002699a

Feasibility of in vivo ${ }^{15} \mathrm{~N}$ MRS detection of hyperpolarized

${ }^{15} \mathrm{~N}$ labeled choline in rats

Cristina Cudalbu, Arnaud Comment, Fiodar Kurdzesau,

Ruud B. van Heeswijk, Kai Uffmann, Sami Jannin, Vladimir

Denisov, Deniz Kirik and Rolf Gruetter, Phys. Chem. Chem.

Phys., 2010, 12, 5818

http://dx.doi.org/10.1039/c002309b

Polychlorinated trityl radicals for dynamic nuclear polarization: the role of chlorine nuclei

Juan Carlos Paniagua, Verónica Mugnaini, Cristina Gabellieri, Miguel Feliz, Nans Roques, Jaume Veciana and Miquel Pons, Phys. Chem. Chem. Phys., 2010, 12, 5824 http://dx.doi.org/10.1039/c003291n

Shuttle DNP spectrometer with a two-center magnet Alexander Krahn, Philip Lottmann, Thorsten Marquardsen, Andreas Tavernier, Maria-Teresa Türke, Marcel Reese, Andrei Leonov, Marina Bennati, Peter Hoefer, Frank Engelke and Christian Griesinger, Phys. Chem. Chem. Phys., 2010, 12,5830

http://dx.doi.org/10.1039/c003381b

Properties of dinitroxides for use in dynamic nuclear polarization (DNP)

Cédric Ysacco, Egon Rizzato, Marie-Alice Virolleaud, Hakim Karoui, Antal Rockenbauer, François Le Moigne, Didier Siri, Olivier Ouari, Robert G. Griffin and Paul Tordo, Phys.

Chem. Chem. Phys., 2010, 12, 5841

http://dx.doi.org/10.1039/c002591g 
Pushing the limit of liquid-state dynamic nuclear polarization at high field

J. A. Villanueva-Garibay, G. Annino, P. J. M. van Bentum and A. P. M. Kentgens, Phys. Chem. Chem. Phys., 2010, 12,5846 http://dx.doi.org/10.1039/c002554m

Solid-state dynamic nuclear polarization at $263 \mathrm{GHz}$ : spectrometer design and experimental results Melanie Rosay, Leo Tometich, Shane Pawsey, Reto Bader, Robert Schauwecker, Monica Blank, Philipp M. Borchard, Stephen R. Cauffman, Kevin L. Felch, Ralph T. Weber, Richard J. Temkin, Robert G. Griffin and Werner E. Maas, Phys. Chem. Chem. Phys., 2010, 12, 5850 http://dx.doi.org/10.1039/c003685b

\section{Resolution and polarization distribution in cryogenic} DNP/MAS experiments

Alexander B. Barnes, Björn Corzilius, Melody L. MakJurkauskas, Loren B. Andreas, Vikram S. Bajaj, Yoh Matsuki, Marina L. Belenky, Johan Lugtenburg, Jagadishwar R. Sirigiri, Richard J. Temkin, Judith Herzfeld and Robert G. Griffin, Phys. Chem. Chem. Phys., 2010, 12, 5861 http://dx.doi.org/10.1039/c003763j

Application of ex situ dynamic nuclear polarization in studying small molecules

Christian Ludwig, Ildefonso Marin-Montesinos, Martin G. Saunders, Abdul-Hamid Emwas, Zoe Pikramenou, Stephen P. Hammond and Ulrich L. Günther, Phys. Chem. Chem. Phys., 2010, 12, 5868

http://dx.doi.org/10.1039/c002700f

${ }^{2} \mathrm{H}$-DNP-enhanced ${ }^{2} \mathrm{H}-{ }^{13} \mathrm{C}$ solid-state NMR correlation spectroscopy

Thorsten Maly, Loren B. Andreas, Albert A. Smith and Robert G. Griffin, Phys. Chem. Chem. Phys., 2010, 12, 5872

http://dx.doi.org/10.1039/c003705b

Thermoresponsive, spin-labeled hydrogels as separable DNP polarizing agents

Björn C. Dollmann, Matthias J. N. Junk, Michelle Drechsler, Hans W. Spiess, Dariush Hinderberger and Kerstin Münnemann, Phys. Chem. Chem. Phys., 2010, 12, 5879 http://dx.doi.org/10.1039/c003349a
A dedicated spectrometer for dissolution DNP NMR spectroscopy

James Leggett, Robert Hunter, Josef Granwehr, Rafal

Panek, Angel J. Perez-Linde, Anthony J. Horsewill, Jonathan McMaster, Graham Smith and Walter Köckenberger, Phys.

Chem. Chem. Phys., 2010, 12, 5883

http://dx.doi.org/10.1039/c002566f

Optimization of dynamic nuclear polarization experiments in aqueous solution at $15 \mathrm{MHz} / 9.7 \mathrm{GHz}$ : a comparative study with DNP at $140 \mathrm{MHz} / 94 \mathrm{GHz}$ Maria-Teresa Türke, Igor Tkach, Marcel Reese, Peter Höfer and Marina Bennati, Phys. Chem. Chem. Phys., 2010, 12, 5893

http://dx.doi.org/10.1039/c002814m

Water ${ }^{1} \mathrm{H}$ relaxation dispersion analysis on a nitroxide radical provides information on the maximal signal enhancement in Overhauser dynamic nuclear polarization experiments

Marina Bennati, Claudio Luchinat, Giacomo Parigi and Maria-Teresa Türke, Phys. Chem. Chem. Phys., 2010, 12, 5902

http://dx.doi.org/10.1039/c002304n

Dynamic nuclear polarization-enhanced solid-state NMR spectroscopy of GNNQQNY nanocrystals and amyloid fibrils

Galia T. Debelouchina, Marvin J. Bayro, Patrick C. A. van der Wel, Marc A. Caporini, Alexander B. Barnes, Melanie Rosay, Werner E. Maas and Robert G. Griffin, Phys. Chem. Chem. Phys., 2010, 12, 5911 http://dx.doi.org/10.1039/c003661g

A $200 \mathrm{GHz}$ dynamic nuclear polarization spectrometer Brandon D. Armstrong, Devin T. Edwards, Richard J. Wylde, Shamon A. Walker and Songi Han, Phys. Chem. Chem. Phys., 2010, 12, 5920 http://dx.doi.org/10.1039/c002290j 


\title{
Shuttle DNP spectrometer with a two-center magnet $\uparrow$
}

\author{
Alexander Krahn, ${ }^{a}$ Philip Lottmann, ${ }^{b}$ Thorsten Marquardsen, ${ }^{a}$ Andreas Tavernier, ${ }^{a}$ \\ Maria-Teresa Türke, ${ }^{b}$ Marcel Reese, ${ }^{b}$ Andrei Leonov, ${ }^{b}$ Marina Bennati, ${ }^{b}$ \\ Peter Hoefer, ${ }^{a}$ Frank Engelke ${ }^{* a}$ and Christian Griesinger ${ }^{* b}$
}

\author{
Received 18th February 2010, Accepted 5th May 2010 \\ First published as an Advance Article on the web 11th May 2010 \\ DOI: $10.1039 / \mathbf{c 0 0 3 3 8 1 b}$
}

\begin{abstract}
A DNP set-up is described where a liquid sample is hyperpolarized by the electron-nucleus Overhauser effect in a field of $0.34 \mathrm{~T}$ and transferred to a field of $14.09 \mathrm{~T}$ for NMR detection. In contrast to a previous set-up, using two dedicated magnets for polarization and detection, a dedicated ferroshim system was inserted into the bore of a $14.09 \mathrm{~T}$ shielded cryomagnet to provide a homogeneous low-field region in the stray field above the magnetic center. After polarization in the low-field the sample is transferred to the high-field magnetic center within $40 \mathrm{~ms}$ by a pneumatic shuttle system. In our set-up a standard high-resolution inverse ${ }^{1} \mathrm{H} /{ }^{13} \mathrm{C}$ selective probe was used for NMR detection and a homebuilt EPR cavity, operating in the $\mathrm{TM}_{110}$ mode was used for polarisation. First experimental data are presented. We observed a maximum proton Overhauser enhancement of up to $\varepsilon^{\mathrm{HF}}=-3.7$ in the high-field position for a $5 \mathrm{mM}$ 4-Oxo-TEMPO-D ${ }^{15} \mathrm{~N}$ (TEMPONE) $/ \mathrm{H}_{2} \mathrm{O}$ sample. While this reproduces the DNP enhancement observed also in the old set-up, with the new set-up we observe enhancement on larger molecules that were impossible to enhance in the old set-up. Therefore, we can demonstrate for the first time Overhauser enhanced high resolution proton spectra of glucose and 2,2-dimethyl-2silapentane-5-sulfonic acid sodium salt (DSS) in $\mathrm{D}_{2} \mathrm{O}$, where the high resolution spectrum was acquired in the high-field position after polarizing the sample in the low-field.
\end{abstract}

\section{Introduction}

Dynamic nuclear polarization (DNP) is a method to increase the nuclear magnetization of samples by a transfer of polarization from unpaired electron spins. DNP can be useful for increasing the sensitivity of nuclear magnetic resonance (NMR) experiments. ${ }^{1-10}$ Due to the high gyromagnetic ratio of the electron its Larmor frequency at $B_{0}$ fields used for high resolution NMR is on the order of several hundred GHz. Set-ups exist where the polarization and the detection take place in the same high static field..$^{2,4,6,11}$ However, the need for high power microwave sources and the high dielectric losses of liquid samples in this frequency range renders these experiments difficult. As an alternative, the sample may be polarized in a lower field where (i) larger sample amounts and (ii) standard EPR equipment can be used, and where (iii) a high DNP coupling factor $\xi$ can be expected for the models of intermolecular motion given in ref. 8 and 12

Over the last two decades, several DNP set-ups have been described where a liquid sample was polarized in a lower external field and afterwards transferred into a higher field for NMR detection. ${ }^{1,7,13}$ In some of these set-ups, the sample is polarized in a frozen state, melted, and transferred to the

\footnotetext{
${ }^{a}$ Bruker BioSpin, Silberstreifen 4, D-76287 Rheinstetten, Germany. E-mail: Frank.Engelke@bruker.de

${ }^{b}$ Max Planck Institute for Biophysical Chemistry, Am Fassberg 11, D-37077 Göttingen, Germany.E-mail: cigr@nmr.mpibpc.mpg.de $\dagger$ Electronic supplementary information (ESI) available: Technical information about the DNP probe, low-field DNP enhancements and high- and low-field longitudinal relaxation rates of the polarized compounds. See DOI: 10.1039/c003381b
}

NMR magnet where a high resolution NMR spectrum is measured at room temperature. ${ }^{1}$ In the latter case, the polarization transfer is based on the solid effect or the thermal mixing effect being effective at low temperatures and for solid samples only. ${ }^{14,15}$ The samples have to be frozen and thawed up which may not be easy for many biological systems. For liquid samples the effective DNP process is the Overhauser effect $^{16}$ that can be efficiently used to directly polarize the nuclear spins when a small amount of a stable radical is dissolved. ${ }^{8}$ In all these set-ups a fast sample transfer between the polarizing field and the detection field is required to avoid a loss of the hyperpolarisation. Fast transfer is mandatory because at low fields the sample experiences a large relaxation rate. Sample shuttling has been extensively used for NMR relaxometry. ${ }^{17,18}$ The shuttle set-up allows for repetition of the experiment and works always under near-physiological conditions of a sample in aqueous solution.

In a previously described prototype that was developed during the work in the Bio-DNP-EU project ${ }^{13}$ an EPR magnet for polarisation was placed on top of the NMR magnet for detection. Because of this construction the field at the sample position fell to $4.5 \mathrm{mT}$ during the sample transfer. As expected and experimentally verified, proton relaxation of large molecules is so fast at this low-field that even with a short shuttle transfer time $t_{\mathrm{sd}}<200 \mathrm{~ms}$ any DNP enhancement obtained at $0.34 \mathrm{~T}$ is partially or completely lost during the shuttle process. ${ }^{13}$ In our novel set-up we seek to minimize the relaxation induced loss of hyperpolarisation during the sample transfer by modifying the field profile so that the magnetic field does not drop below the field strength of $0.34 \mathrm{~T}$ at which hyperpolarisation occurs. 
We achieve this by polarizing the sample in the stray field of the 14.1 T NMR magnet, $470 \mathrm{~mm}$ above the high-field (HF) position. As a result, the spins experience a monotonically increasing static field as the sample travels from the low-field to the high-field position.

The signal enhancement factor at low-field (LF) $\varepsilon^{\mathrm{LF}}$ caused by the Overhauser effect is commonly expressed by the DNP coupling factor $\xi$, the leakage factor $f$, and the saturation factor $s$ as ${ }^{8}$

$$
\varepsilon^{\mathrm{LF}}=\frac{\left\langle I_{\mathrm{DNP}}\right\rangle}{\left\langle I_{\mathrm{no} \mathrm{DNP}}\right\rangle}=1+\xi f s \frac{\gamma_{\mathrm{S}}}{\gamma_{\mathrm{I}}}
$$

where the saturation factor $s$ describes the degree to which the electron spin transitions are saturated. After the shuttle process, taking the high-field Boltzmann polarization as a reference and assuming pure dipolar coupling (i.e. $\xi=0.5$ ), the maximum enhancement factor can be written as

$$
\varepsilon_{\mathrm{LF}}^{\mathrm{HF}}=\frac{B_{0, \mathrm{EPR}}}{B_{0, \mathrm{NMR}}}\left(1+\frac{\gamma_{\mathrm{S}}}{2 \gamma_{\mathrm{I}}}\right)
$$

where $B_{0, \mathrm{EPR}}$, and $B_{0, \mathrm{NMR}}$ are the static field values at the polarization and detection position, and $\gamma_{\mathrm{S}}$ and $\gamma_{\mathrm{I}}$ are the gyromagnetic ratios of the electron and the proton, respectively.

To perform shuttle DNP experiments, a relatively high technical effort is necessary, and therefore it appears worthwhile to describe our set-up in more detail. In the next chapter all relevant spectrometer components are outlined. Special emphasis is put on the shuttle system and on a novel compact microwave cavity that facilitates a fast sample transfer in the magnet bore. In chapter 3.1, we describe the experimental optimization procedure for shuttle DNP experiments. Finally, in chapter 3.2, we present preliminary results in which we observe a polarization transfer to target biomolecules dissolved in water. With our improved set-up, we observe for the first time a hyperpolarized high resolution proton spectrum on glucose with an enhancement factor of up to -2.8 . Such enhancements could not be observed with the previous design with two separate magnets. Therefore, we owe it to the here described novel set-up that we can observe solution DNP of biomolecules.

\section{DNP shuttle spectrometer}

\subsection{Overview}

Our shuttle DNP spectrometer consists of a $14.1 \mathrm{~T}$ standard bore $(54 \mathrm{~mm})$ shielded cryomagnet (Bruker $600 \mathrm{MHz}$ US), with an extra ferroshim system installed in the stray field at $0.34 \mathrm{~T}, 470 \mathrm{~mm}$ above the magnetic center (Fig. 1). The measured field profile along the magnet axis featuring a monotonically increasing field along the shuttle path is illustrated in Fig. 2. For NMR detection a Bruker Avance console and a modified high resolution NMR probe are used. In the low-field plateau, a compact cavity resonator is installed and tuned to the corresponding electron Larmor frequency of $\nu_{2} \approx 9.5 \mathrm{GHz}$. The cavity is connected to a Bruker $E$-Scan EPR spectrometer that also drives additional sweep and modulation coils being installed in the ferroshim system and in the cavity, respectively. A cylindrical sample container can be pneumatically moved between the cavity and the NMR probe. ${ }^{13}$

A typical timing diagram for our shuttle spectrometer is illustrated in Fig. 3. During the polarization time $t_{\mathrm{p}}$ the sample is located in the low-field position inside the cavity and is irradiated at the electron spin Larmor frequency. The duration $t_{\mathrm{p}}$ of the microwave irradiation is determined by the polarization build-up time (see below). After the shuttle down time $t_{\mathrm{sd}}$ the sample arrives in the NMR probe and the signal is measured after an additional post-shuttle delay $t_{\mathrm{psd}}$. In our set-up, the pneumatic shuttle system and the gate of the microwave amplifier are triggered by the NMR console and therefore the optimum polarization time and the pre- and post-shuttle delays can be controlled by the pulse program. The shuttle time depends on the applied pressure. It was found to be $t_{\mathrm{sd}}=40 \mathrm{~ms}$ in our experiments with a pressure reading of 4 bar. In the next section, the spectrometer and its components will be described in more detail.

\subsection{The ferroshim system}

The ferroshim system is composed of a ferromagnetic cylinder inserted into the magnet bore from the top of the magnet. ${ }^{5}$ It has been optimized to compensate for the static field gradient at a position where the stray field has a static induction of approximately $0.34 \mathrm{~T}$. With the ferroshim it is possible to get a homogeneous region with an axial length of $10 \mathrm{~mm}$. The homogeneity of the field plateau is calculated with $\pm 1 \mathrm{mT}$. The position of the ferroshim system inside the magnet bore can be adjusted by $\pm 5 \mathrm{~mm}$ in axial direction to obtain a field plateau between $0.325-0.360 \mathrm{~T}$ corresponding to electron and proton spin Larmor frequencies in the range 9.19-10.17 $\mathrm{GHz}$ and $13.96-15.46 \mathrm{MHz}$, respectively. The measured field profile along the magnet axis with the installed ferroshim system and a LF plateau at $0.34 \mathrm{~T}$ is shown in Fig. 2. In addition to the ferroshim system, an additional sweep coil $\left(Z_{0}\right)$ and a shim coil $\left(Z_{1}\right)$ are installed. The latter is driven by a direct current sourceł and allows to further improve the field homogeneity in the plateau by producing a gradient with a strength of $2.82 \pm 0.02 \mathrm{mT} / \mathrm{cm}^{-1} \mathrm{~A}^{-1}$. With the sweep coil an extra field of $5.24 \pm 0.002 \mathrm{mT} \mathrm{A}^{-1}$ can be superimposed to the static field. A sweep current of approximately $\pm 1 \mathrm{~A}$ is applied by the $E$-Scan console that results in a maximum spectral width of $104 \mathrm{G}(10.4 \mathrm{mT})$ over the EPR spectrum.

\subsection{DNP cavity}

In the bore of the ferroshim system a probe head featuring a cylindrical cavity operating at $9.5 \mathrm{GHz}$ in the $\mathrm{TM}_{110}$ mode is installed. In this mode the microwave magnetic field $B_{1}^{\mathrm{MW}}$ is oriented orthogonal to the common longitudinal axis of the cavity and the magnet. $B_{1}^{\mathrm{MW}}$ shows no variation along the axial cavity length. Hence, this mode is especially suitable for a cylindrical sample placed concentrically inside the cavity. The modal fields in a transverse plane of a cylindrical cavity are shown in Fig. 4. In addition, the electric microwave field along the longitudinal magnet axis is zero and small in the close vicinity of the axis, as is required by the orthogonality of the

$\ddagger$ Here we used a stand-alone Bruker BSMS shim unit connected to the host computer. 


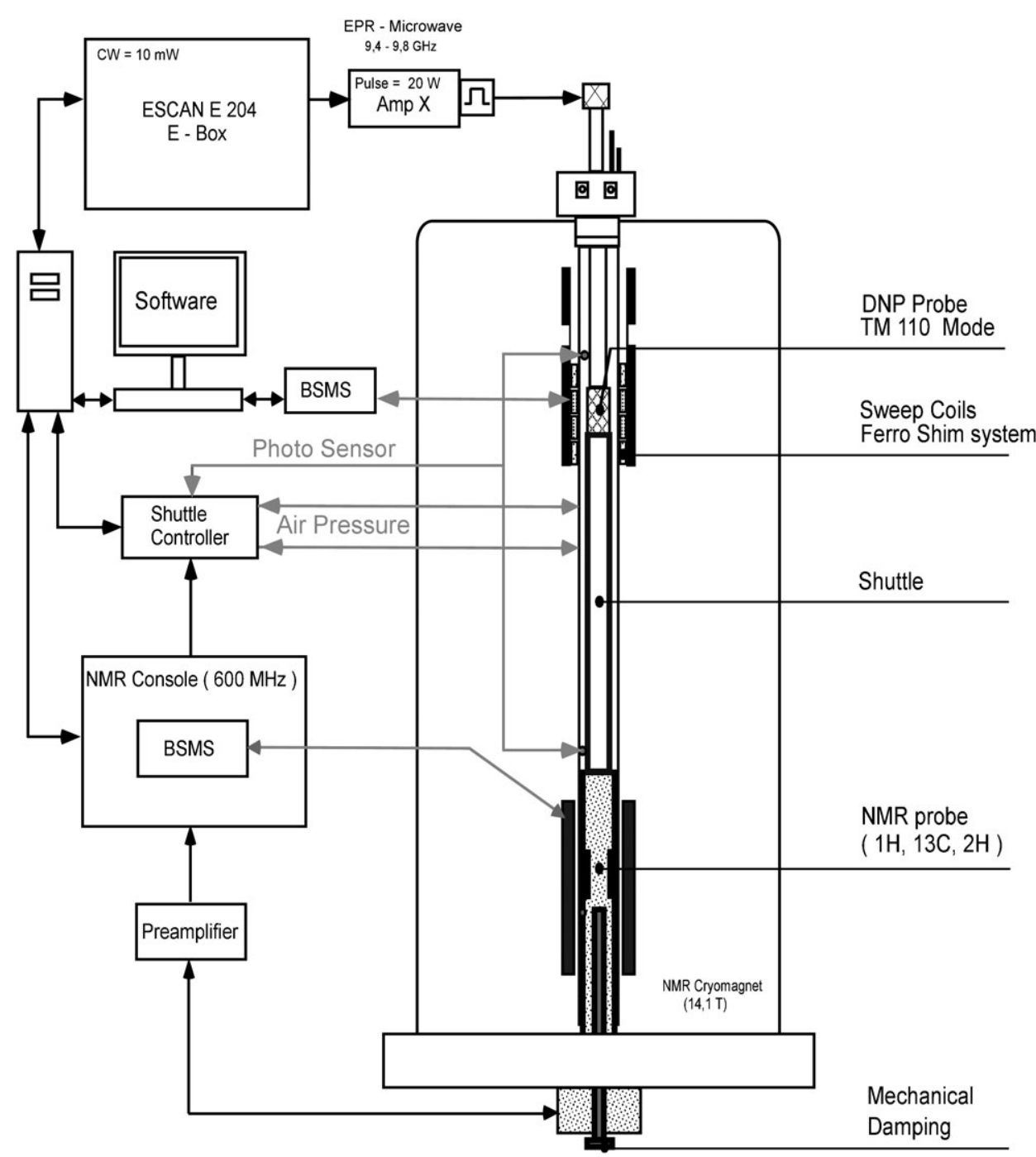

Fig. 1 Overview over the shuttle DNP spectrometer. The system consists of a Bruker $600 \mathrm{MHz}$ US magnet with an additional ferroshim system installed in the stray field and that provides an additional field plateau above the high-field magnetic center (low-field position). In this plateau a microwave cavity is installed that is tuned to the corresponding electron Larmor frequency and connected to a Bruker E-Scan EPR console. The console is used either to measure the EPR line from a sample placed inside the cavity (for this purpose the console also controls the currents in a sweep coil and in a modulation coil - for details see text) or to drive a microwave amplifier for microwave cw irradiation. The sample is placed inside a shuttle container that can be pneumatically transferred between the cavity in the low-field position and a modified NMR probe at the high-field position in the magnetic center.

modal fields. In our set-up, the inner diameter of the shim system of $40 \mathrm{~mm}$ restricts the available space for the cavity. Considering a cavity body with a wall thickness of $4 \mathrm{~mm}$ the maximum inner cavity diameter is $32 \mathrm{~mm}$. For an empty cylindrical cavity, the resonance frequency of the $\mathrm{TM}_{110}$ mode is determined only by the inner diameter as

$$
f_{\mathrm{r}}=\frac{c_{0} a_{m n}}{\pi D}
$$

where $c_{0}$ is the speed of light, $a_{m n}$ is the $n$-th zero of the $m$-th order Bessel function (i.e. $a_{11}=3.832$ ), and $D$ is the inner cavity diameter. ${ }^{19,20}$ Hence, the available diameter would result in a resonance frequency of $f_{\mathrm{r}}=11.4 \mathrm{GHz}$. To tune the cavity to the desired frequency we used a concentric PTFE ring of appropriate inner and outer diameter (for details see below). In addition, a quartz tube was installed coaxially inside the cavity volume that extends through the top and bottom plates and so allows the sample to enter the resonator volume. The cavity was coupled to the microwave source by a standard UT141 coaxial cable with a short stub extending into the resonator volume. By altering the penetration depth of the stub, critical coupling can be obtained. Moreover, to measure an EPR signal, a modulation field $\mathbf{B}_{\text {mod }}$ parallel to the direction of the static magnetic field is required. This field is applied by a pair of Helmholtz coils embedded into the top and bottom plates of the cavity body. To allow the modulation field to enter the cavity volume the top and bottom plates were made of Vespel with a thin sheet (thickness $\approx 35 \mu \mathrm{m}$ ) of copper foil that is transparent for the modulation field. Each modulation coil has an inner diameter of $25 \mathrm{~mm}$ and is wound with 100 turns. The optimization of the cavity resonator was performed in two steps, as described in the following. 


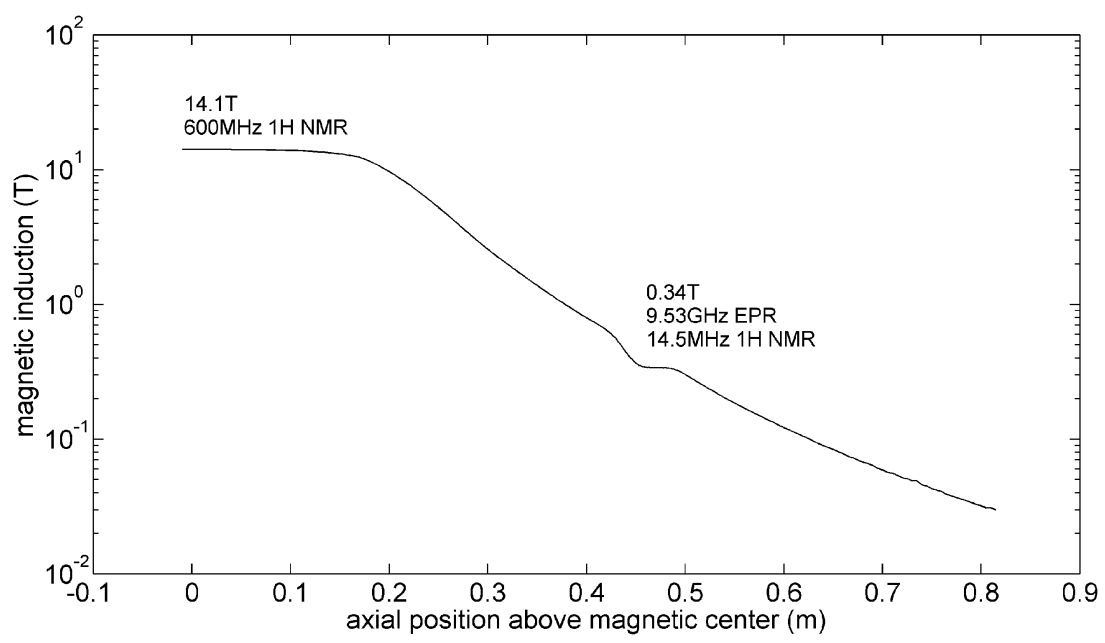

Fig. 2 Measured magnetic field profile along the magnet bore of a Bruker US $600 \mathrm{MHz}$ magnet where an additional ferroshim system is installed $470 \mathrm{~mm}$ above the magnetic center. With this shim system an extra plateau is obtained in the stray field above the magnetic center that can be adjusted between 0.328 and $0.363 \mathrm{~T}$ corresponding to an electron Larmor frequency between 9.19 and $10.17 \mathrm{GHz}$.

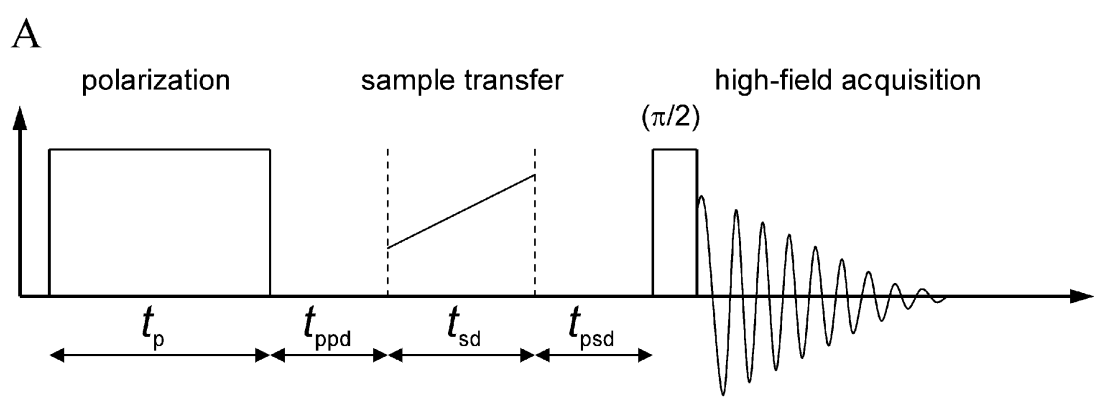

B

polarization low-field acquisition

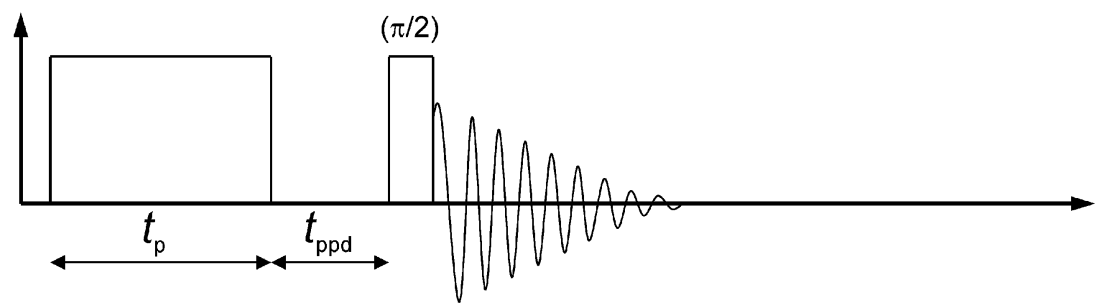

Fig. 3 (A) Characteristic timing diagram for shuttle DNP experiments. During the polarization build-up time $t_{\mathrm{p}}$ the sample is placed in the low-field position and irradiated with a microwave field at the electron spin Larmor frequency. After the post-polarisation delay $t_{\mathrm{ppd}}$ and during the shuttle down time $t_{\mathrm{sd}}$ the sample is transferred to the high-field position into the NMR probe where the signal is measured after the post-shuttle delay $t_{\mathrm{psd}}$ by the application of a detection pulse. (B) In one version of our set-up, the enhanced signal can be directly measured in the low-field position. For this purpose, an additional NMR coil is installed inside the cavity and tuned to the low-field proton-Larmor frequency.

Firstly, we used a radial mode matching (RMM) technique to calculate the dimensions of the dielectric ring and to estimate the losses due to an aqueous sample, and secondly, the finite integration technique was employed to calculate the characteristics of the final design. Due to the overall cylindrical symmetry of the cavity resonator, the RMM technique can be employed which allows an efficient calculation of the microwave electromagnetic field distribution ${ }^{21}$ in cylindrical cavities. For a cavity with an inner diameter of $32 \mathrm{~mm}$ and an axial length of $20 \mathrm{~mm}$ we calculated the appropriate dimensions of the dielectric ring for a target $\mathrm{TM}_{110}$ resonance frequency of 9.5 GHz. In the calculation all materials were considered as lossless (perfect electric conductors (PEC) and real valued permittivities). Nevertheless, the resonator $\mathrm{Q}$ factor can be estimated when the dielectric losses in the sample and the PTFE ring are considered as perturbation to the ideal field. For the PTFE material we assumed a relative permittivity of $\varepsilon_{\mathrm{r}}=2.08$ and a loss tangent of $\tan \delta=0.0004$. Taking into account also the quartz shuttle guide $\left(\varepsilon_{\mathrm{r}}=3.78\right.$, with an inner and outer diameter of $3.5 \mathrm{~mm}$ and $5 \mathrm{~mm}$ ) we chose 17 and $23 \mathrm{~mm}$ as the inner and outer diameter for the PTFE ring, respectively, to obtain a $\mathrm{TM}_{110}$ resonance frequency of $9.512 \mathrm{GHz}$. The calculated overall cavity $\mathrm{Q}$ factor was $\mathrm{Q}=3188$ where we considered a wall conductivity similar 


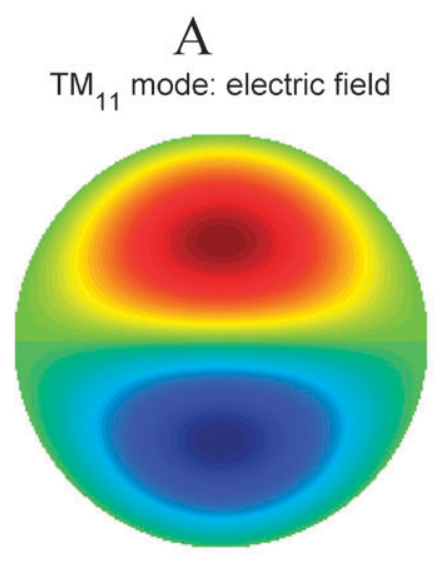

$\mathrm{C}$

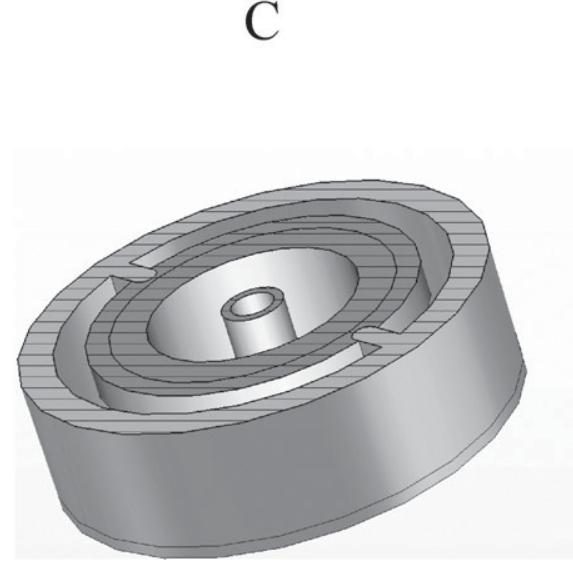

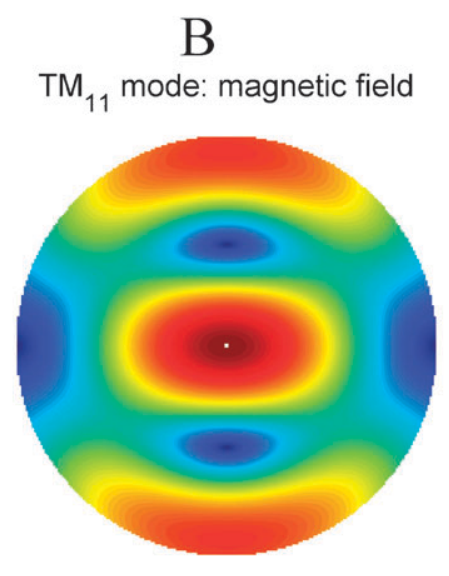

D

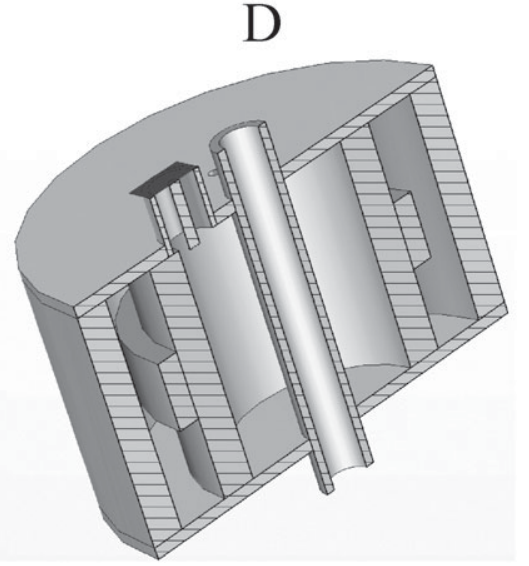

Fig. 4 (A + B) Magnetic and electric modal field distribution of the $\mathrm{TM}_{11}$ mode in a transversal plane of the cylindrical waveguide. The electric field is zero along the horizontal axis whereas the magnetic field is maximum around the center (details see text). (C) Simulation model of the DNP cavity with two plates to direct the $\mathrm{TM}_{110}$ modal field direction and the dielectric ring. (D) The same resonator geometry with the coupling structure modelled for a numerical electromagnetic field analysis.

to copper $\left(\sigma=58 \times 10^{6} \mathrm{~S} \mathrm{~m}^{-1}\right)$. With the same method the influence of the shuttle container with a lossy sample can be estimated. Modelling the shuttle container as a quartz tube with an inner diameter of $0.75 \mathrm{~mm}$ filled with an aqueous sample $\left(\tan \delta=0.4936\right.$ at $9.5 \mathrm{GHz}$ and $T=25{ }^{\circ} \mathrm{C}$ ) we calculated a Q factor of 2552 and a frequency of $9.498 \mathrm{GHz}$. The dielectric constant of water was found from a first order Debye model ${ }^{22}$

$$
\varepsilon=\varepsilon_{0}+\frac{\varepsilon_{0}-\varepsilon_{\infty}}{1+\omega^{2} \tau^{2}}
$$

where $\varepsilon_{0}=78$ and $\varepsilon_{\infty}=4$ are the static and high frequency permittivity of pure water and $\tau=8.84 \times 10^{-12} \mathrm{~s}$ is the relaxation time. ${ }^{23}$ Although the RMM method is useful for fast estimation of the correct cavity dimensions and sample induced losses, it can only be applied when an overall cylindrical geometry is maintained.

Alternatively, numerical methods can be used when the cylindrical symmetry is broken. In the following, we have employed the finite integration technique (FIT) implemented into a commercial software package (Microwave studio-CST Darmstadt). In a first step we have added two extra plates to enforce a specific direction for the $\mathrm{TM}_{110}$ magnetic field and a thin additional PTFE support for the dielectric ring (Fig. 4C). With these changes and using a dedicated Eigenmode solver, the calculated resonance frequency of the empty cavity shifted to $f_{\mathrm{r}}=9.425 \mathrm{GHz}$ with an unloaded Q factor of 3090 . For comparison, the calculation was repeated with a shuttle container and an aqueous sample as described above, where we obtained a resonance at $f_{\mathrm{r}}=9.413 \mathrm{GHz}$ with a Q of 2540 . Note the good agreement with the results from the RMM method above.

Finally, we set up a simulation with a transient solver to take into account the coupling structure and to calculate nearby spurious modes. Two spurious modes are present in the frequency range of interest $(9-10 \mathrm{GHz}$ ) (Fig. S1), $\dagger$ where in particular a spurious hybrid mode at $9.52 \mathrm{GHz}$ is close to the desired target frequency.

For our first experiments, it was desirable to probe the initial enhancement factor compared to Boltzmann polarization in the low-field position (prior to a sample transfer). Therefore an additional RF saddle coil was arranged around the quartz tube inside the cavity. In doing so, special care was taken to minimize the interference between the RF coil and the microwave field. In theory, interference free arrangement of the saddle coil with respect to the microwave field is obtained when the RF magnetic field is orthogonal to the microwave field and when the coil wire sections that are oriented parallel to the cavity axis are placed close to each other along the $x$ axis (where the electric microwave field is zero). 
In our set-up, the absorption of microwave energy by the liquid sample limits the maximum sample diameter. From the field calculations we found that the ratio between the sample radius and the free space wavelength should be less than 0.01 . Here, this corresponds to a sample diameter of approximately $0.7 \mathrm{~mm}$.

\subsection{Sample shuttle system and NMR probe}

During the sample transfer the sample container is guided inside a metal tube that connects the quartz glass guides in the probe and in the cavity. The motion of the shuttle is controlled by a home built microprocessor board that drives the pneumatic valves. To detect the arrival of the shuttle container two light barriers are installed at the end positions whose signals are detected by the shuttle controller. With this system we measured a total shuttle time of less than $50 \mathrm{~ms}$ for the $470 \mathrm{~mm}$ between the two plateaus when an air pressure of approximately 3.5 bar is applied. To accelerate thermal sample equilibration between the experiments a constant flow of shuttle gas is applied around the shuttle container at both end positions.

The NMR shuttle probe is a modified high resolution probe (dual ${ }^{1} \mathrm{H} /{ }^{13} \mathrm{C}$ with ${ }^{2} \mathrm{H}$ lock channel) (Fig. 5A). Inside the probe an extension of the shuttle tube towards the lower end facilitates the insertion of the sample into the shuttle system without removing the probe (Fig. 1). The basic mechanical design of the probe is equivalent to a normal $5 \mathrm{~mm}$ highresolution probe where low-susceptibility and/or susceptibility compensated materials are used. This is especially important for the NMR coil wire material and all other components in close vicinity of the coil. Here the susceptibility compensation is optimized for an operation under nitrogen atmosphere. As a result we obtain the same spectral resolution after shimming as for standard high resolution probes. The RF circuit corresponds to that of a typical dual-probe with an inner saddle coil for ${ }^{1} \mathrm{H}$ and ${ }^{13} \mathrm{C}$ observation, and an orthogonal outer coil for an additional ${ }^{2} \mathrm{H}$ lock channel (Fig. 5).

Due to the high speed of the shuttle container, special care has to be taken to prevent mechanical vibrations of the probe that can lead to noticeable artefacts in the spectrum. Therefore, the shuttle system is additionally mechanically decoupled from the probe body to minimize vibrations and to achieve the necessary reproducibility for the shuttle DNP experiments.

To improve the sensitivity of the NMR measurement a new shuttle container (Fig. 5B) was designed with a reduced outer diameter in combination with a tightly wound detection coil. The body of the container is a synthetic quartz glass tube (Suprasil, inner diameter $0.76 \mathrm{~mm}$, outer diameter $3.26 \mathrm{~mm}$ ) with Vespel plugs on both ends. The sample volume is sealed by toroidal fluoropolymer joints in the end caps. An active sample length of $12 \mathrm{~mm}$ (limited by the length of the detection coil) corresponds to an active sample volume of $6 \mu \mathrm{l}$. The chosen wall thickness is sufficient to withstand the mechanical stress during the shuttle process.

\section{Experimental}

In order to estimate the performance of the new set-up as compared to the old one ${ }^{13}$ we used the same sample compositions and determined the optimal operational parameters for our new set-up. These include the optimal polarization time $t_{\mathrm{p}}$ for a given radical concentration, the microwave power, and the minimum post-shuttle delay $t_{\mathrm{psd}}$. The latter is necessary to allow a mechanical settling of the sample.

In a second step we conducted shuttle DNP experiments in which we measured Overhauser enhanced proton spectra for small organic molecules. While such experiments did not show a signal enhancement with the old set-up, we now observe a substantial signal enhancement with the new system. We attribute this to the novel magnet set-up that reduces relaxation losses during the sample transfer by a shorter shuttle distance and a monotonously increasing field.

We used the nitroxide radicals 4-hydroxy-TEMPO- ${ }^{14} \mathrm{~N}$ (TEMPOL) and 4-oxo-TEMPO-D,${ }^{15} \mathrm{~N}$ (TEMPONE) as polarizer like in the former set-up. ${ }^{10,13,24}$ Based on previous experience we chose a polarizer concentration of $5 \mathrm{mM}$ to avoid larger relaxation losses caused by higher concentrations between the polarization step and NMR measurement in the low-field. ${ }^{25}$ For an accurate determination of the sample temperature we added DSS to our samples as an internal standard. Its chemical shift is nearly constant over a wide range of temperatures. We used the fitting functions for the temperature dependent chemical shift of water given in ref. 26 to calculate the sample temperature and to determine the microwave induced sample heating.

For polarizing small organic molecules we chose ethanol, DSS and glucose. Ethanol is easy to polarize and shows only modest relaxation losses during shuttling. The multiplet structure of the peaks gives first hints about the resolution that can be expected for the enhanced proton signals. Glucose is a larger molecule and therefore it shows larger relaxation losses as compared to water, ethanol and DSS. Therefore it is a crucial test for the potential of the novel spectrometer design.

\subsection{Set-up of experiments}

In a first set of experiments the basic functionality of the low-field set-up was tested by measuring the amount of hyperpolarisation in the low-field position. In these experiments the cavity was tuned to a microwave frequency of $9.6 \mathrm{GHz}$ with the internal saddle coil tuned to the nuclear Larmor frequency. A shuttle container with an active sample volume of $9.8 \mu \mathrm{l}$ (inner diameter $1 \mathrm{~mm}$, active length $12.5 \mathrm{~mm}$ ) filled with $10 \mathrm{mM}$ TEMPOL- ${ }^{14} \mathrm{~N} / \mathrm{H}_{2} \mathrm{O}$, compromising three EPR lines, was used for these experiments. The corresponding EPR spectrum consists of three lines.

The axial position of the cavity in the ferroshim system was fine-adjusted by optimizing the line width in the TEMPOL- ${ }^{14} \mathrm{~N}$ EPR spectrum. In a similar manner the residual $B_{0}$ inhomogeneity was compensated with an appropriate current in the $Z_{1}$ shim coil. To determine the optimal sweep field position a microwave pulse with $t_{\mathrm{p}}=1 \mathrm{~s}$ was applied at a frequency of $9.58 \mathrm{GHz}$ with a microwave power of $1.9 \mathrm{~W}$ prior to the NMR detection (Fig. 3B). The resulting NMR signal was compared with the reference signal from a single pulse experiment without microwave irradiation. The obtained enhancement factor as the amplitude ratio of the hyperpolarized signal and the reference signal is displayed in Fig. S2A. $\dagger$ 


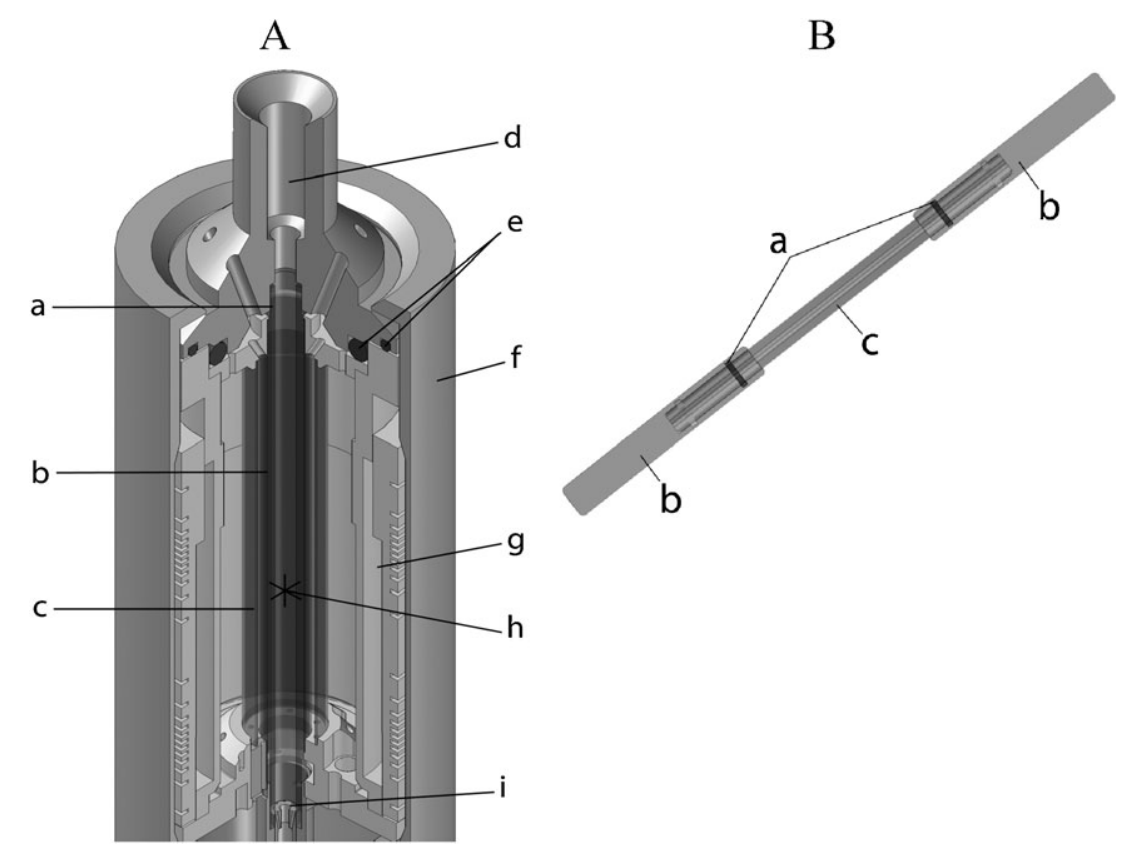

Fig. 5 (A) Details of the NMR probe with the glass tube (a) to position the shuttle container in the NMR coil (b $+\mathrm{c})$ in the magnetic center (h). The connection to the transfer system is made via a conical adaptor (d) that is supported by rubber toroidal joints to attenuate mechanical vibrations (e). The stopper (i) for the shuttle consists of an air jet that applies the pressure to shuttle the sample to the low-field position and that provides a laminar stream of shuttle gas when the sample is in the NMR probe. An additional $Z$-gradient coil (g) is installed inside the probe cover (f) for future experiments. (B) Geometry of the shuttle container where the capillary (c) is terminated with two end caps (b) and sealed with two toroidal gaskets.

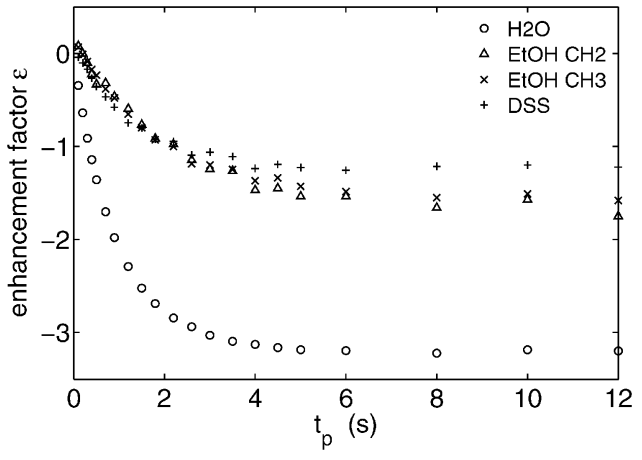

Fig. 6 Enhancement build-up curve for a sample of $5 \mathrm{mM}$ TEMPONE-D, ${ }^{15} \mathrm{~N}, 10 \mathrm{mM}$ DSS and $0.1 \mathrm{M}$ EtOH in $80 / 20 \mathrm{H}_{2} \mathrm{O} / \mathrm{D}_{2} \mathrm{O}$ (11 mm sample length) measured at the high-field position after a variable polarization time $t_{\mathrm{p}}$ with a fixed microwave power of $15 \mathrm{~W}$. Note that these results are analogous to the ones illustrated in Fig. S2C where the polarization build-up time was measured in the low-field with the additional saddle coil.

With these values for $t_{\mathrm{p}}$, and $P_{\mathrm{MW}}$ the maximum enhancement factor was $\varepsilon^{\mathrm{LF}}=-15$ when the sweep field was adjusted for a microwave irradiation on one of the three hyperfine lines. With a microwave irradiation at the center line the dependence of the enhanced signal amplitude on the microwave pulse power was measured (Fig. S2B). $\dagger$ For an available microwave power of $9 \mathrm{~W}$ a maximum low-field enhancement factor of $\varepsilon^{\mathrm{LF}} \approx-60$ was achieved. For a single homogeneously broadened EPR line the inverse saturation factor is proportional to the inverse polarization power, and the enhancement for total saturation can be estimated by extrapolation to infinite power. ${ }^{12}$ In our case, the microwave field is irradiated only on one TEMPOL- ${ }^{14} \mathrm{~N}$ EPR line and therefore other effects such as Heisenberg exchange or temperature effects may be present that lead to a more subtle power dependence. For a microwave power of $P_{\mathrm{MW}}=1.9 \mathrm{~W}$ the decay of the hyperpolarized signal in the low-field is observed by incrementing the variable delay $t_{\text {ppd }}$ after the microwave pulse (Fig. S2C). $\dagger$ Finally, the minimal length for the microwave pulse was determined by subsequently increasing the polarization time (Fig. S2D). $\dagger$ For a pulse length of $>1$ s no further polarization increase was observed.

Summarizing the results of the TEMPOL- ${ }^{14} \mathrm{~N}$ low-field experiments the optimum sample polarization is achieved when the sweep field is set such that the microwave field is on-resonance with one of the TEMPOL- ${ }^{14} \mathrm{~N}$ hyperfine lines within an accuracy of 1-2 G. For further experiments the internal saddle coil was removed to improve the cavity $\mathrm{Q}$ factor and to reduce distortions of the microwave field within the sample volume.

\subsection{Shuttle DNP-first results}

In the following we will describe how we proceeded from polarization of water to the polarization of larger biologically relevant molecules like glucose. As explained in the following, we observe in the new set-up similar enhancements for water as we did in the old set-up. This is due to the fact that water is a small molecule and therefore does not relax efficiently during shuttling. Glucose, however, could not be polarized in the old set-up. The best we ever reached was a DNP signal that was -0.3 times the Boltzman signal. At the end of this paragraph, 
we report that with the new set-up, relaxation can be minimized such that an enhancement of -2.8 is observed.

We first set out to recapitulate the results obtained for water in the old set-up now in the new set-up. In order to evaluate whether the new set-up was superior we added a larger molecule (DSS) to the solution. Molecules larger than water could not be polarized in the old set-up. We therefore used a $5 \mathrm{mM}$ TEMPONE-D, ${ }^{15} \mathrm{~N}$ solution $\left(80 / 20 \mathrm{H}_{2} \mathrm{O} / \mathrm{D}_{2} \mathrm{O}\right)$ with $5 \mathrm{mM}$ DSS as standard sample with an axial length of $11 \mathrm{~mm}$ limited by UV-glue plugs. ${ }^{15} \mathrm{~N}$ labeled TEMPONE has only two hyperfine lines instead of three as in unlabeled nitroxide radicals. Due to the fact that the microwave field can be irradiated on-resonance with only one of the hyperfine lines, a higher saturation factor ${ }^{9}$ can be reached when using the ${ }^{15} \mathrm{~N}$ labelled sample as compared to the ${ }^{14} \mathrm{~N}$ isotopomer. ${ }^{15} \mathrm{~N}$ labeled TEMPONE was synthesized as described in ref. 3 .

In our experiments the shuttle time was $t_{\mathrm{sd}}=37 \mathrm{~ms}$, which is a reduction to $1 / 3$ as compared to the old set-up. ${ }^{13}$ After sample arrival an additional delay of $t_{\mathrm{psd}}=70 \mathrm{~ms}$ was necessary due to post-arrival jumps and vibrations of the shuttle container. Upon arrival, the shuttle container was repelled. The concomitant jumps were detected with an optical sensor at the end position. While these jumps occurred only for a few ms, reproducible NMR measurements were possible only after a total delay of $70 \mathrm{~ms}$. With the pressure and driving gas $\left(\mathrm{N}_{2}\right)$ currently available it is not possible to further reduce the shuttle time significantly. In all experiments - excepting low-field relaxation - the post polarization delay $t_{\text {ppd }}$ was set to $0 \mathrm{~ms}$.

We used the low-field EPR line for saturation and optimized the sweep field value by a set of short shuttle-DNP experiments. In these experiments, the $B_{0}$ field was set to the low-field line and changed in steps of $0.4 \mathrm{G}$ until maximum enhancement was observed. For this optimization procedure a short irradiation time of $1 \mathrm{~s}$ at a low microwave power of $10 \mathrm{~W}$ was sufficient. In the same manner as for the low-field experiments we recorded a field sweep over both hyperfine lines (Fig. S3). $\dagger$

As above, we used for the first shuttle experiments a $5 \mathrm{mM}$ TEMPONE-D,${ }^{15} \mathrm{~N}$ solution $\left(80 / 20 \mathrm{H}_{2} \mathrm{O} / \mathrm{D}_{2} \mathrm{O}\right)$ with $10 \mathrm{mM}$ DSS and $0.1 \mathrm{M}$ ethanol. The enhancement as a function of polarization time is illustrated in Fig. 6. After approximately $4 \mathrm{~s}$ maximum enhancement is reached for water, ethanol and DSS. The enhancement is negative implying that the (dominating) polarization transfer is due to a dipolar coupling to the electrons, and that the hyperpolarisation can be preserved during the shuttle process (Fig. 7). The maximum enhancement factors for ethanol were measured as $\varepsilon^{\mathrm{HF}}=-1.7$ calculated from the ratio between the integral of the respective signal with and without DNP. (Table 1).

We observed that for ethanol and DSS the signal enhancement extracted from the amplitude ratio is larger as compared to the ratio extracted from the integrals. This is due to the fact that the reference Boltzmann spectrum was measured at ambient temperature, while the sample temperature after DNP is increased because of microwave heating (also see the companion paper of this issue: Türke, et $a l .{ }^{27}$ ). The higher sample temperature leads to a longer transverse relaxation time of the resonances in the DNP spectrum and therefore to larger
Table 1 Measured enhancement factors of Fig. 7 with $t_{\mathrm{p}}=12 \mathrm{~s}$ and the spin lattice relaxation time $T_{1}$ of the low- and high-field positions for a sample of $5 \mathrm{mM}$ TEMPONE-D, ${ }^{15} \mathrm{~N}$ solution $\left(80 / 20 \mathrm{H}_{2} \mathrm{O} / \mathrm{D}_{2} \mathrm{O}\right)$ with $10 \mathrm{mM}$ DSS and $0.1 \mathrm{M}$ ethanol

\begin{tabular}{lllll}
\hline Region/ppm & & $\varepsilon^{\mathrm{HF}}$ & $T_{1}^{\mathrm{LF}} / \mathrm{s}$ & $T_{1}^{\mathrm{HF}} / \mathrm{s}$ \\
\hline $4.80 \ldots 4.40$ & $\mathrm{H}_{2} \mathrm{O}$ & -3.2 & 1.0 & 1.8 \\
$3.60 \ldots 3.50$ & $\mathrm{CH}_{3}-\mathrm{CH}_{2}-\mathrm{OH}$ & -1.6 & 1.5 & 2.3 \\
$1.15 \ldots 1.05$ & $\mathrm{CH}_{3}-\mathrm{CH}_{2}-\mathrm{OH}$ & -1.8 & 1.6 & 2.4 \\
$-0.05 \ldots-0.10$ & $\mathrm{DSS}$ & -1.2 & 0.9 & 1.4 \\
\hline
\end{tabular}

intensities. Thus, the enhancement measured on the integral is lower than that measured on the amplitude. On the contrary, the chemical shift of the water protons is temperature dependent and the inhomogeneity of the temperature causes line broadening of the water peak. Here, we measured temperature gradients of up to $16{ }^{\circ} \mathrm{C}$ across the sample.

Alternatively, we can measure the DNP spectrum and the reference spectrum at the same elevated temperature. Then the integral of the reference spectrum will decrease leading to a larger integral enhancement factor $\left(\varepsilon^{\mathrm{HF}}\right)$. At the same time, the reference spectrum will have sharper lines leading to an increase of the signal intensities. This reduces the enhancement factor measured on the signal amplitude $\varepsilon_{\mathrm{amp}}^{\mathrm{HF}}$ when DNP and reference spectra are taken at the same temperature. Taking the DNP enhanced and the reference spectra at the same temperature leads to a convergence of the two methods to quantify the enhancement (Table 2).

Furthermore, we characterized the proton $T_{1}$ relaxation under inversion recovery at high-field (Fig. S4). $\dagger$

In addition, the low- and high-field spin-lattice relaxation time $T_{1}$ could be measured by varying the post-polarization delay $t_{\text {ppd }}$ after the microwave irradiation, and the post-shuttle delay $t_{\mathrm{psd}}$ after the sample arrival in the high-field position (Fig. S5). $\dagger$ The corresponding pulse sequence is shown in Fig. 3A. Fig. S5A illustrates the decay of the hyperpolarized signal towards the Boltzmann polarization in the high-field position when varying the delay $t_{\mathrm{ppd}}$ while keeping the shuttle time and the post shuttle delay $t_{\mathrm{psd}}$ constant. $\dagger$ Note that for long values of $t_{\mathrm{ppd}}$ the initial hyperpolarized magnetization has already decayed towards the low-field Boltzmann value before the shuttle process. In our measurements we find after the shuttling $\mathrm{ca} .10 \%$ of the value typically expected for the highfield position. We attribute this to an additional build-up of magnetization during the shuttle motion. Without this additional build-up the polarization expected should only be $2.5 \%$ ( $1 / 40$ equal to the ratio of the static fields for EPR and NMR). Fig. S5B in contrast shows the polarization decay when setting $t_{\mathrm{ppd}}$ to zero, keeping the shuttle time constant and varying $t_{\mathrm{psd}} \cdot \dagger$

Table 2 Measured enhancement factors dependent on the temperature $\left(T_{\mathrm{B}}\right)$, i.e. the temperature for the measurement of the Boltzmann signal without DNP. Sample $5 \mathrm{mM}$ TEMPONE-D, ${ }^{15} \mathrm{~N}$ solution $(80 / 20$ $\left.\mathrm{H}_{2} \mathrm{O} / \mathrm{D}_{2} \mathrm{O}\right)$ with $10 \mathrm{mM}$ DSS and $0.1 \mathrm{M}$ ethanol

\begin{tabular}{lllllllll}
\hline$T_{\mathrm{B}} /{ }^{\circ} \mathrm{C}$ & $\begin{array}{l}20 \\
\varepsilon^{\mathrm{HF}}\end{array}$ & $\begin{array}{l}20 \\
\varepsilon_{\mathrm{amp}}^{\mathrm{HF}}\end{array}$ & $\begin{array}{l}25 \\
\varepsilon^{\mathrm{HF}}\end{array}$ & $\begin{array}{l}25 \\
\varepsilon_{\mathrm{amp}}^{\mathrm{HF}}\end{array}$ & $\begin{array}{l}30 \\
\varepsilon^{\mathrm{HF}}\end{array}$ & $\begin{array}{l}30 \\
\varepsilon_{\mathrm{amp}}^{\mathrm{HF}}\end{array}$ & $\begin{array}{l}35 \\
\varepsilon^{\mathrm{HF}}\end{array}$ & $\begin{array}{l}35 \\
\varepsilon_{\mathrm{amp}}^{\mathrm{HF}}\end{array}$ \\
\hline $\mathrm{H}_{2} \mathrm{O}$ & -3.2 & & -3.3 & & -3.4 & & -3.6 & \\
$\mathrm{CH}_{3}-\mathrm{CH}_{2}-\mathrm{OH}$ & -1.6 & -2.2 & -1.6 & -2.0 & -1.7 & -1.6 & -1.8 & -1.6 \\
$\mathrm{CH}_{3}-\mathrm{CH}_{2}-\mathrm{OH}$ & -1.8 & -2.4 & -1.8 & -2.3 & -1.9 & -2.0 & -2.0 & -1.8 \\
$\mathrm{DSS}$ & -1.2 & -1.7 & -1.3 & -1.6 & -1.3 & -1.3 & -1.4 & -1.3 \\
\hline
\end{tabular}




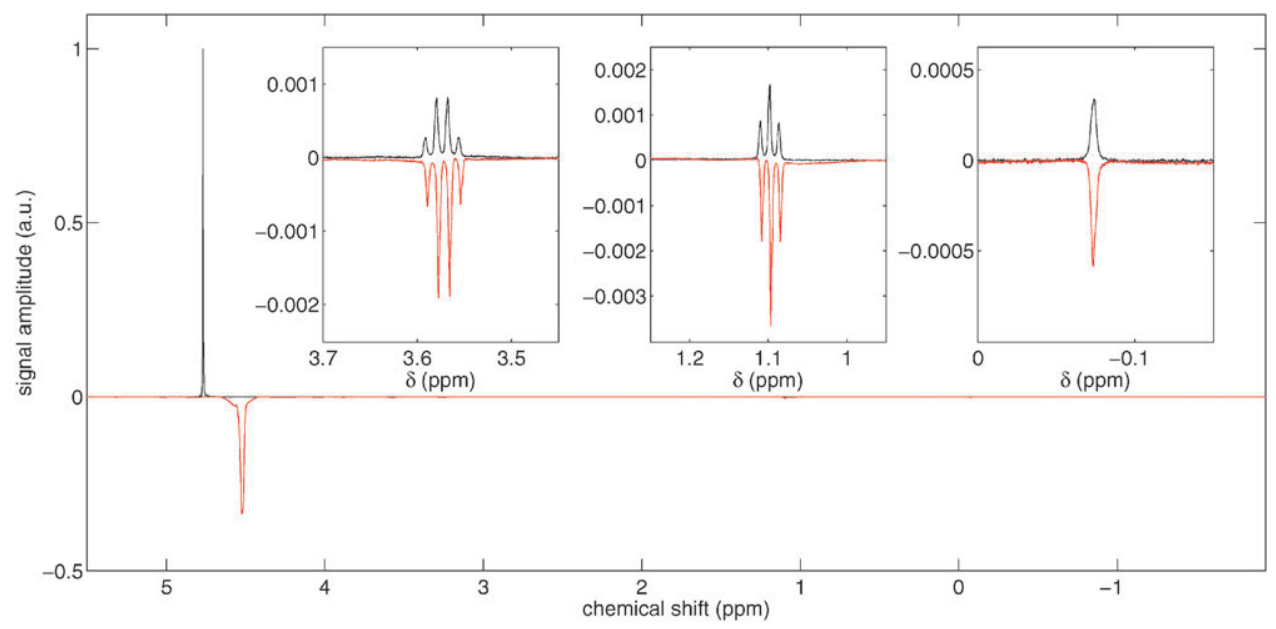

Fig. 7 Proton spectrum of ethanol at room temperature (black) vs. the DNP enhanced spectrum (red) of $0.1 \mathrm{M}$ ethanol $80 / 20 \mathrm{H}_{2} \mathrm{O} / \mathrm{D}_{2} \mathrm{O}$ with $5 \mathrm{mM}$ TEMPONE-D, ${ }^{15} \mathrm{~N}$ and $10 \mathrm{mM}$ DSS solution. The reference signal was measured with a standard experiment consisting of a short $\pi / 2$ deg pulse with duration of $7.1 \mu \mathrm{s}$. For the DNP experiment the sample was shuttled from the high-field to the low-field position. The microwave was irradiated for $12 \mathrm{~s}$ with a power of $16 \mathrm{~W}$. Directly after the microwave irradiation $\left(t_{\mathrm{ppd}}=0 \mathrm{~s}\right)$, the sample was shuttled to the high-field position $\left(t_{\mathrm{s}}=40 \mathrm{~ms}\right)$ and after a settling time of $t_{\mathrm{psd}}=70 \mathrm{~ms}$, the detection pulse was applied. Note that in addition to the $\mathrm{H}_{2} \mathrm{O}$ proton also the proton signals from the ethanol and the DSS reference show a negative enhancement. The shift and broadening of the water peak are caused by microwave heating. For the selected regions, the enhancement factors determined from the integrals are given in Table 1.

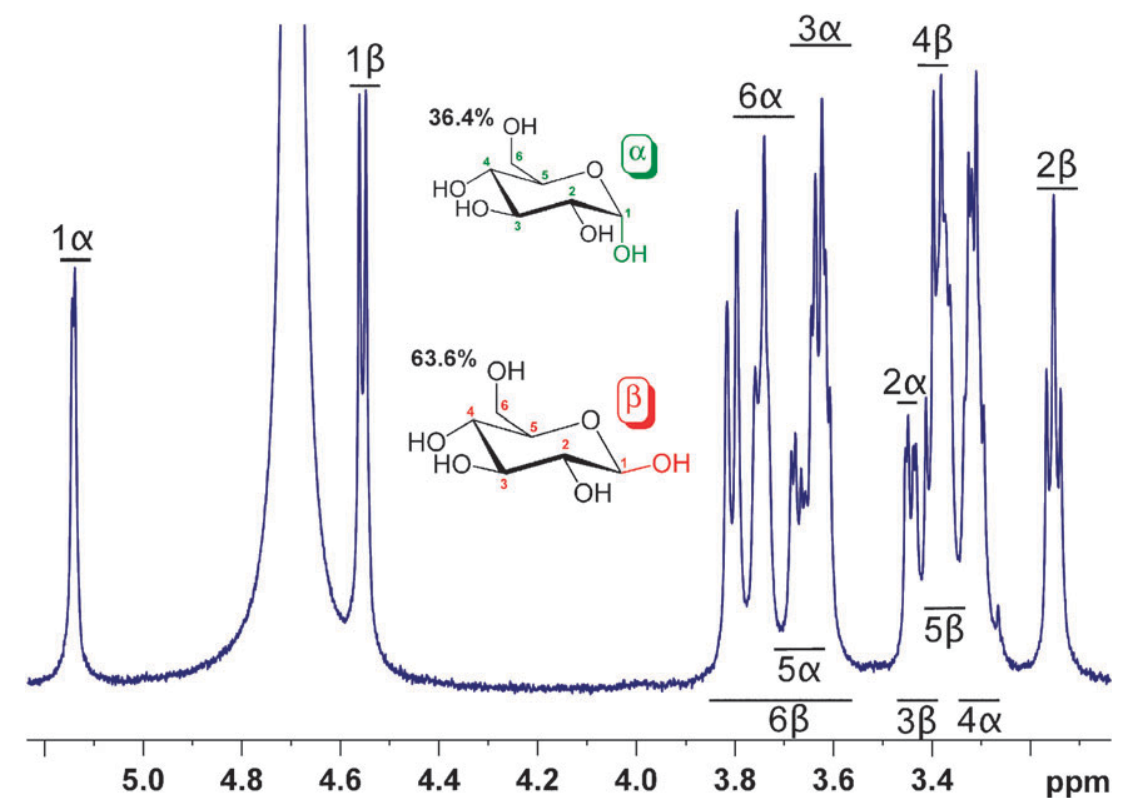

Fig. 8 Boltzmann reference spectrum at $26.9^{\circ} \mathrm{C}$ of D-glucopyranose in $\mathrm{D}_{2} \mathrm{O}$ with the schematic mapping of the peaks for the $\alpha$ and $\beta$ anomers. ${ }^{28}$ The sample consisted of $0.5 \mathrm{M}$ D-glucose in $99.8 \% \mathrm{D}_{2} \mathrm{O}$ with $10 \mathrm{mM}$ TEMPONE-D,${ }^{15} \mathrm{~N}$ and $5 \mathrm{mM}$ DSS.

The low-field and the high-field $T_{1}$ times constitute the upper and lower limits of the relaxation rates during the shuttle motion, with the low-field rate being larger than the high-field rate. Since the timing after the DNP is known, the relaxation losses can be estimated. Using this way of calculating the range of relaxation losses, we find for water using the sum of $t_{\mathrm{sd}}$ and $t_{\text {psd }} 7-10 \%$, for ethanol $7-9 \%$ and for DSS $13-15 \%$.

Encouraged by the unprecendently low relaxation losses observed in the new set-up, we went one step further and polarized glucose. We used a $10 \mathrm{mM}$ TEMPONE-D, ${ }^{15} \mathrm{~N}$ solution in $99.8 \% \mathrm{D}_{2} \mathrm{O}$ with $5 \mathrm{mM}$ DSS and $0.5 \mathrm{M}$ D-glucose
(Figs. 8 and 9). The water, DSS and the D-glucose protons show a negative enhancement. We achieved an enhancement factor in the range of $\varepsilon^{\mathrm{HF}}=-1.4$ to -2.8 for the different protons of the D-glucose (Table 3, Fig. 7). From the low-field and high-field $T_{1}$ relaxation shown in Fig. S6† we anticipate relaxation losses for the glucose protons between 10 and $30 \%$. From this last measurement, we owe it to the new set-up with short shuttle distance that relaxation can be suppressed to a degree that we can enhance molecules considerably larger than water. This opens the door to biomolecular enhancement in solution by Overhauser effect based DNP. 

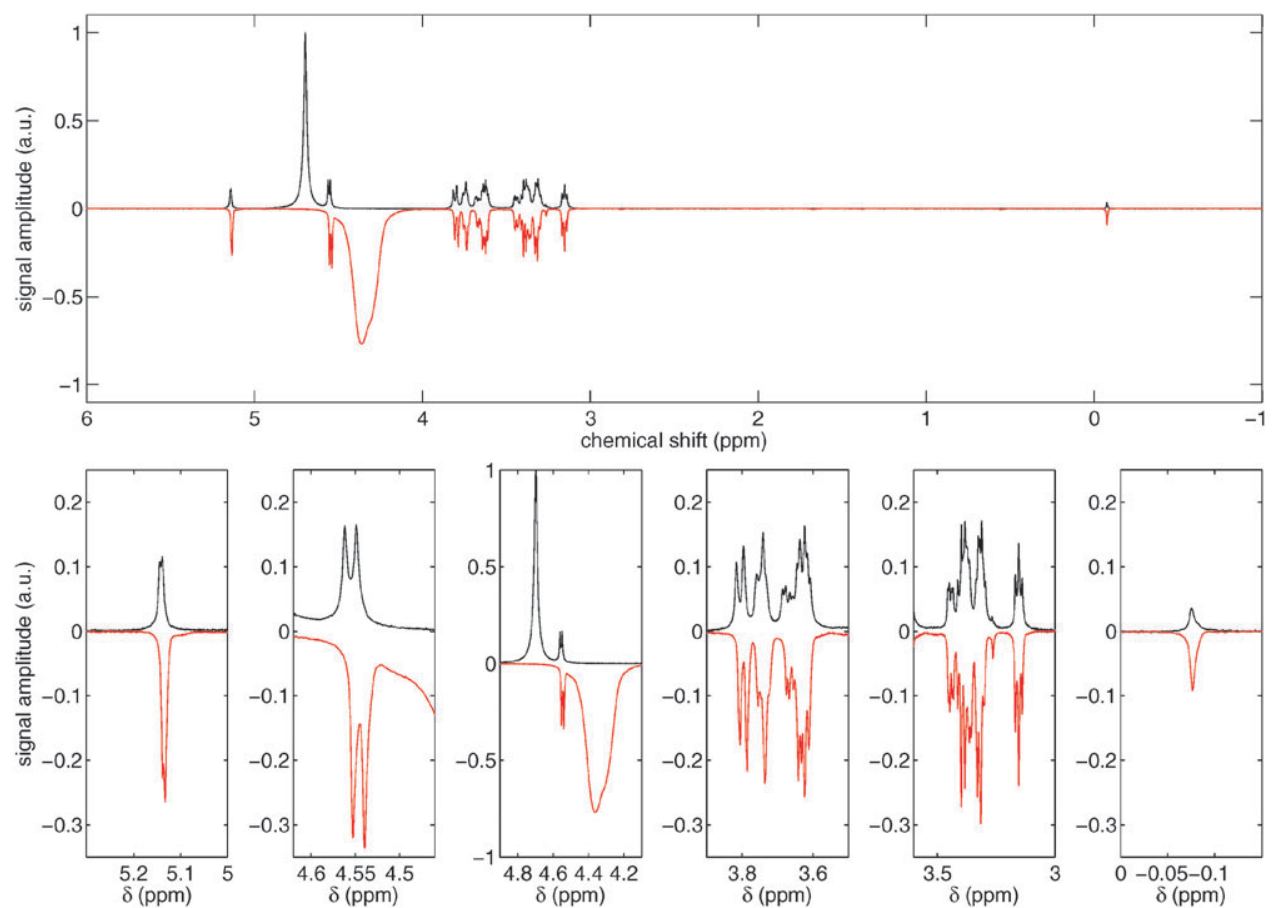

Fig. 9 Proton spectrum of glucose at $26.9{ }^{\circ} \mathrm{C}$ (black) vs. the DNP enhanced spectrum at $60.3{ }^{\circ} \mathrm{C}$ (red) of $0.5 \mathrm{M}$ D-glucose in $99.8 \% \mathrm{D}_{2} \mathrm{O}$ with $10 \mathrm{mM}$ TEMPONE-D, ${ }^{15} \mathrm{~N}$ and $5 \mathrm{mM}$ DSS solution. The reference signal was measured with a standard experiment consisting of a short $\pi / 2$ deg pulse with a duration of $7.2 \mu \mathrm{s}$. For the DNP experiment the sample was shuttled from the high-field to the low-field position. After a short delay $(\sim 2 \mathrm{~s})$ to allow the sample to settle, the microwave was irradiated for $5 \mathrm{~s}$ with a power of $20 \mathrm{~W}$. Directly after the microwave irradiation $\left(t_{\mathrm{ppd}}=0 \mathrm{~s}\right)$, the sample was shuttled to the high-field position $\left(t_{\mathrm{sd}}=40 \mathrm{~ms}\right)$ and after a settling time of $t_{\mathrm{psd}}=70 \mathrm{~ms}$, the detection pulse was applied. Note that in addition to the $\mathrm{H}_{2} \mathrm{O}$ proton, also the proton signals from the glucose and the DSS reference show a negative enhancement. The shift and broadening of the water peak are caused by microwave heating (under the described conditions a temperature increase of approximately $35 \mathrm{~K}$ with a $16 \mathrm{~K}$ gradient can be estimated from the chemical shift of the water peak). For the selected regions, the enhancement factors determined from the integrals $\varepsilon^{\mathrm{HF}}$ are given in Table 3 .

Table 3 Enhancement factors as determined from the integral values of the selected signal regions in Fig. 8

\begin{tabular}{lll}
\hline Region/ppm & Groups & $\varepsilon^{\mathrm{HF}}$ \\
\hline $5.30 \ldots 5.00$ & D-glycopyranose $1 \alpha$ & -2.2 \\
$4.60 \ldots 4.50$ & D-glycopyranose $1 \beta$ & -2.8 \\
$5.00 \ldots 4.10$ & $\mathrm{H}_{2} \mathrm{O}$ & -3.7 \\
$3.90 \ldots 3.55$ & D-glycopyranose $3 \alpha+5 \alpha+6 \alpha+6 \beta$ & -1.4 \\
$3.50 \ldots 3.10$ & D-glycopyranose $2 \alpha+4 \alpha+2 \beta+3 \beta+4 \beta+5 \beta$ & -1.5 \\
$0.10 \ldots-0.30$ & DSS & -2.0 \\
\hline
\end{tabular}

\section{Conclusions and outlook}

In conclusion we show that proton enhancements for biologically relevant molecules considerably larger than water were achieved. The fact that glucose can be enhanced shows the qualitative difference of the new set-up as compared to the old set-up. This is mainly due to the fact that with the new set-up relaxation losses have been reduced by shuttling the sample from the field that is used for DNP to the high-field detection position without passing through an extremely low-field region. The next step is to further improve the experimental parameters for obtaining maximum enhancement at low-field and to study the relevance of other pathways that may reduce the enhancement, such as NOE between $\mathrm{H}_{2} \mathrm{O}$ and the molecule we want to polarize. In addition, we intend to further investigate DNP enhancement on hetero-nuclei such as
${ }^{15} \mathrm{~N}$ and ${ }^{13} \mathrm{C}$, which appeared very promising already with our previous set-up. We are confident that with the new set-up shuttle DNP with unfolded and globular proteins is within reach.

\section{Acknowledgements}

We gratefully acknowledge fruitful discussions with Marian Kloza and Ion Prisecaru (Bruker Biospin, Rheinstetten) for the design of the microwave cavity and with Pierre-Alain Bovier, Robert Schauwecker, Thomas Speck, Daniel Eckert and Dirk Wilhelm (Bruker Biospin, Fällanden) who constructed the ferroshim system. This work was funded by the EU through the BIO-DNP: Dynamic Nuclear Polarization for NMR in Structural Biology. Additional funding was received from the Max Planck Gesellschaft (to M.B. and C.G.). 


\section{References}

1 J. H. Ardenkjaer-Larsen, B. Fridlund, A. Gram, G. Hansson, L. Hansson, M. H. Lerche, R. Servin, M. Thaning and $\mathrm{K}$. Golman, Increase in signal-to-noise ratio of $>10000$ times in liquid-state NMR, Proc. Natl. Acad. Sci. U. S. A., 2003, 100(18), 10158-10163.

2 V. S. Bajaj, C. T. Farrar, M. K. Hornstein, I. Mastovsky, J. Bryant, K. E. Kreischer, R. J. Temkin and R. G. Griffin, Dynamic nuclear polarization at $9 \mathrm{~T}$ using a novel $250 \mathrm{GHz}$ gyrotron microwave source, J. Magn. Reson., 2003, 160(2), 85.

3 B. A. Bates, M. E. Johnson and B. L. Currie, Stable isotope substituted spin labels. 2. An improved synthesis of perdeuterio${ }^{15} \mathrm{~N}$-(1-oxyl-2,2,6,6-tetramethyl-4-piperidinyl)maleimide, J. Labelled Compd. Radiopharm., 1983, 20(1), 33-38.

4 L. R. Becerra, G. J. Gerfen, B. F. Bellew, J. A. Bryant, S. J. Hall, D. A. Inati, R. T. Weber, S. Un, T. F. Prisner, A. E. McDermott, K. W. Fishbein, K. E. Kreischer, R. J. Temkin, D. J. Singel and R. G. Griffin, A spectrometer for dynamic nuclear polarization and electron paramagnetic resonance at high frequencies, J. Magn. Reson. Ser. A, 1995, 117, 28-40.

5 P.-A. Bovier, R. Schauwecker, D. Eckert, Apparatus for executing DNP-NMR measurements with compensation assembly and method for aligning the compensation assembly. Patent EP2146215A1.

6 V. Denysenkov, M. Prandolini, A. Krahn, M. Gafurov, B. Endeward and T. F. Prisner, High field DNP spectrometer for liquids, Appl. Magn. Reson., 2008, 34, 289-299.

7 S. Grosse, F. Gubaydullin, H. Scheelken, H. M. Vieth and A. V. Yurkovskaya, Field cycling by fast NMR probe transfer: Design and application, Appl. Magn. Reson., 1999, 17(2-3), 211-225.

$8 \mathrm{~K}$. H. Hausser and D. Stehlik, Dynamic nuclear polarization in liquids, Adv. Magn. Reson., 1968, 3, 79-139.

9 P. Höfer, P. Carl, G. Guthausen, T. Prisner, M. Reese, T. Carlomagno, C. Griesinger and M. Bennati, Studies of dynamic nuclear polarization with nitroxides in aqueous solution, Appl. Magn. Reson., 2008, 34, 393-398.

10 P. Höfer, G. Parigi, C. Luchinat, P. Carl, G. Guthausen, M. Reese, T. Carlomagno, C. Griesinger and M. Bennati, Field dependent dynamic nuclear polarization with radicals in aqueous solution, J. Am. Chem. Soc., 2008, 130, 3254-3255.

11 M. J. Prandolini, V. P. Denysenkov, M. Gafurov, S. Lyubenova, B. Endeward, M. Bennati and T. F. Prisner, First DNP results from a liquid water-tempol sample at $400 \mathrm{MHz}$ and $260 \mathrm{GHz}$, Appl. Magn. Reson., 2008, 34, 399-407.

12 W. Müller-Warmuth and K. Meise-Gresch, Molecular motions and interactions as studied by dynamic nuclear polarization (DNP) in free radical solutions, Adv. Magn. Reson., 1983, 11, 1-45.

13 M. Reese, D. Lennartz, T. Marquardsen, P. Höfer, A. Tavernier, P. Carl, T. Schippmann, M. Bennati, T. Carlomagno, F. Engelke and C. Griesinger, Construction of a liquid-state NMR DNP shuttle spectrometer: First experimental results and evaluation of optimal performance characteristics, Appl. Magn. Reson., 2008, 34, 301-311.

14 R. A. Wind, M. J. Duijvestijn, C. van der Lugt, A. Manenschijn and J. Vriend, Applications of dynamic nuclear polarization in ${ }^{13} \mathrm{C}$ NMR in solids, Prog. Nucl. Magn. Reson. Spectrosc., 1985, 17, 33-67.

15 W. T. Wenckebach, The solid effect, Appl. Magn. Reson., 2008, 34(3-4), 227-235.

16 A. W. Overhauser, Polarization of nuclei in metals, Phys. Rev., 1953, 92(2), 411-415.

17 A. G. Redfield, Shuttling device for high-resolution measurements of relaxation and related phenomena in solution at low field, using a shared commercial $500 \mathrm{MHz}$ NMR instrument, Magn. Reson. Chem., 2003, 41(10), 753-768.

18 A. G. Redfield, Hybrid electro-pneumatic sample shuttler for use in a shared commercial high-resolution spectrometer and recent results obtained with it, XXIInd International Conference of Magnetic Resonance in Biological Systems, Göttingen, 2006.

19 K. Simonyi, Theoretische Elektrotechnik, VEB Deutscher Verlag der Wissenschaften, 8 edn, 1980.

20 R. F. Harrington, Time-Harmonic Electromagnetic Fields, Wiley-Interscience, 2001. IEEE Press Classic Reissue.

21 Y. E. Nesmelov, A. Gopinath and D. D. Thomas, Aqueous sample in an EPR cavity: sensitivity considerations, J. Magn. Reson., 2004, 167, 138-146.

22 M. Simeonova and J. Gimsa, The influence of the molecular structure of lipid membranes on the electric field distribution and energy absorption, Bioelectromagnetics, 2006, 27, 652-666.

23 CRC Handbook of Chemistry and Physics, ed. R. C. Weast, M. J. Astle and W. H. Beyer, CRC Press, Boca Raton, Florida, 65th edn, 1984.

24 M. Reese, M. T. Türke, I. Tkach, G. Parigi, C. Luchinat, T. Marquardsen, A. Tavernier, P. Höfer, F. Engelke, C. Griesinger and $\mathrm{M}$. Bennati, ${ }^{1} \mathrm{H}$ and ${ }^{13} \mathrm{C}$ dynamic nuclear polarization in aqueous solution with a two-field $(0.35 \mathrm{~T} / 14 \mathrm{~T})$ shuttle DNP spectrometer, J. Am. Chem. Soc., 2009, 131, 15086-15087.

25 C. Luchinat and G. Parigi, Nuclear relaxometry helps designing systems for solution DNP on proteins, Appl. Magn. Reson., 2008, 34, 379-392.

26 R. E. Hoffman, Standardization of chemical shifts of TMS and solvent signals in NMR solvents, Magn. Reson. Chem., 2006, 44, 606-616.

27 M.-T. Türke, I. Tkach, M. Reese, P. Höfer and M. Bennati, Optimization of dynamic nuclear polarization experiments in aqueous solution at $15 \mathrm{MHz} / 9.7 \mathrm{GHz}$ : a comparative study with DNP at $140 \mathrm{MHz} / 94 \mathrm{GHz}$, Phys. Chem. Chem. Phys., 2010, 12 DOI: $10.1039 / \mathrm{c} 002814 \mathrm{~m}$

28 D. J. Koch and A. S. Perlin, Synthesis and ${ }^{13}$ C NMR spectrum of D-glucose-3- $d$. bond-polarization differences between the anomers of D-glucose, Carbohydr. Res., 1970, 15(3), 403-410. 